\title{
Online support community for adolescents and young adults (AYAs) with cancer: user statistics, evaluation, and content analysis
}

This article was published in the following Dove Press journal:

Patient Preference and Adherence

\author{
Suzanne EJ Kaal',2 \\ Olga Husson ${ }^{2-4}$ \\ Fleur van Dartel ${ }^{3}$ \\ Karin Hermans ${ }^{1,2}$ \\ Rosemarie Jansen ${ }^{1,2}$ \\ Eveliene Manten-Horst ${ }^{1,2}$ \\ Petra Servaes ${ }^{2,3}$ \\ Tom $\mathrm{H}$ van de Belt ${ }^{5}$ \\ Lucien JLPG Engelen ${ }^{5}$ \\ Judith B Prins ${ }^{2,3}$ \\ Suzan Verberne ${ }^{6}$ \\ Winette TA van der \\ Graaf ${ }^{\prime 2,4}$
}

'Department of Medical Oncology, Radboud University Medical Center, Nijmegen, the Netherlands; ${ }^{2}$ Dutch

AYA "Young and Cancer" Platform,

AYA Platform Radboudumc, Radboud University Medical Center, Nijmegen, the Netherlands; ${ }^{3}$ Department of Medical Psychology, Radboud University Medical Center, Nijmegen, the Netherlands; ${ }^{4}$ Division of Clinical Studies, Institute of Cancer Research and Royal Marsden NHS Foundation Trust, London, UK; ${ }^{5}$ Radboud REshape and Innovation Center, Radboud University Medical Center, Nijmegen, the Netherlands; 'Leiden Institute for Advanced Computer Science, Leiden University, Leiden, the Netherlands

Correspondence: Winette TA van der Graaf

Department of Medical Oncology, Radboud University Medical Center, PO Box 910I, 6500 HB Nijmegen, the Netherlands

Tel +3I 2436 I 3457

Email winette.vandergraaf@radboudumc.nl
Purpose: Peer support is an important unmet need among adolescent and young adult (AYA) cancer patients. This study was conducted to describe the use and evaluation of a Dutch secure online support community for AYA diagnosed with cancer between 18 and 35 years.

Methods: User statistics were collected with Google analytics. Community members were asked to complete questionnaires on the usefulness of the community. A content analysis using Linguistic Inquiry and Word Count was conducted.

Results: Between 2010 and 2017, the community received 433 AYA members (71\% female; mean age at diagnosis 25.7 years; 52 Dutch hospitals represented). The mean time since diagnosis when subscribing to the community was 2.7 years (SD 4.4). Questionnaire data among 30 AYA community members indicated that the use of the community resulted in acknowledgment and advice regarding problems (56\%) and the feeling of being supported (63\%). Almost half of the respondents felt less lonely, 78\% experienced recognition in stories of other AYA. Anonymized content analysis $(n=14)$ showed that the majority of the online discussions encompassed emotional and cognitive expressions, and emotional support.

Conclusion: The secure Dutch online AYA community can help AYA cancer patients to express feelings, exchange information, address peer support, and has been found helpful in coping with cancer. As AYA cancer patients often lack the option of meeting each other in person, the AYA community is helpful in establishing peer support. Its use would benefit from promotion by health care professionals.

Keywords: adolescent and young adult, AYA, AYA cancer patient, online community, peer support, user statistics, content analysis

\section{Introduction}

Adolescence and young adulthood are crucial phases with regard to social, professional, physical and psychological development. Having cancer seriously disrupts this development and can have a negative impact on issues regarding control over life, body image, finances, education, work plans, relationships, and plans for having children. ${ }^{1}$ The Dutch definition of AYA (diagnosed with cancer between 18 and 35 years) is based on the organization of the health care system in the Netherlands, in which pediatric oncology, for patients between 0 and 18 years at diagnosis, is centralized and adult oncology is only centralized for rare or complex cancer types. AYA cancer patients cannot profit from integrated care provided by pediatric oncology centers.

AYA cancer patients frequently $(40 \%-50 \%)$ report unmet (supportive) needs including contact with peers, access to age-appropriate information, treatment facilities, emotional support services, and fertility services, ${ }^{2-4}$ thus underlining the fact that 
supportive care for this age group is not optimal to address all age-specific needs. ${ }^{5}$ In response, in the Netherlands and other European countries, initiatives to improve AYA cancer care have started recently. ${ }^{6,7}$

E-Health interventions are increasingly being used in cancer care - for example, to support patients in managing problems in daily life and gaining knowledge. ${ }^{8}$ E-Health can be defined as "information and communication technology, especially the Internet, to improve or enable health and health care". ${ }^{9}$ According to the behavior change model of Ritterband, internet interventions can lead to symptom improvement through a combination of personal and environmental factors on the one hand (eg, knowledge, motivation, and beliefs) and specific website characteristics on the other hand (eg, appearance, content, and delivery). ${ }^{10}$ The Internet can be used by patients to find (medical) information and share stories on weblogs, forums, and online social networks (Facebook, Twitter) and online communities. ${ }^{11}$ Online communities are platforms where individuals meet and exchange experience and information. ${ }^{12}$ Previous studies showed that participating in an online community can have an empowering and therapeutic effect: patients find informational and emotional support, ${ }^{13,14}$ recognition, ${ }^{12,15}$ emotional expression, and insight. ${ }^{16}$ As a technical- and electronics-savvy generation, AYAs are primed to benefit from supportive care delivered through e-Health, alongside usual care. ${ }^{17,18}$ Currently there are six AYA communities/websites with different features to exchange informational, emotional, and social support as described in Table 1. However, none of them provides a secure environment.

Because AYAs with cancer express age-specific peer support as an important unmet need and given that exchange of information can be rather privacy-sensitive, a secure, closed online community could be an asset in addressing this need. ${ }^{18-20}$ In 2010, an online community named AYA4 (All Information You've Asked for) was developed by and for Dutch AYA cancer patients treated at the Radboud University Medical Center (Radboudumc). This online community became available for all AYA cancer patients in the Netherlands in 2014. The aim of this article is to describe how the Dutch online AYA community is currently being used and to evaluate in particular 1) user statistics, 2) usefulness, and 3) content analysis addressing the psychological processes expressed in the messages on the forum.

\section{Methods}

\section{Development of an online AYA community}

The online community for AYA cancer patients was developed in close collaboration between the department of medical oncology, REshape \& Innovation Center, and AYA cancer patients of the Radboudumc. The community works as follows. At first access, a community manager verifies age- and disease-specific information in the treating hospital. A disclaimer explains details of the community: for example, that members have to sign a digital will to define what has to be done with their community content after one's death. The content of the community is only accessible for AYA patients with login details. The only person who has access and is able to communicate with all users is the community manager, a non-health care professional, trained in communication, who is online for approximately 4 hours daily (with a stand-in, in case of absence). When patients log in for the first time, they are welcomed by the community manager and they are notified that she can facilitate forum discussions. She will not share personal information of AYA community members with health care professionals, unless the AYA patients give explicit permission to do so.

\section{Procedure}

The Ethics Committee of the Radboudumc judged that no detailed review was warranted, given the nonintrusive character of this study (\#2016-2872).

\section{Study I: user statistics}

When signing up to the community, patients have to fill out the following information: first name, surname, gender, date of birth, telephone number, email address, treating hospital, patient identification number, treating physician, nurse specialist, type of cancer diagnosis, age at and date of cancer diagnosis, date of start treatment, and what to do with the account in case of death. Logging data (assessed April 11, 2017) about activity and duration of logging in were analyzed using Google Analytics, a web analytics service offered by Google that tracks and records website traffic. Login data of the community manager were excluded from this analysis.

\section{Study 2: evaluation}

The evaluation study of the online AYA community was part of a larger as yet unpublished study aimed to gain insight into the supportive care needs of AYA cancer patients in the Netherlands. Patients aged between 18 and 35 years at the time of cancer diagnosis were invited to participate and were recruited via a website of patient advocates (www. kanker.nl and www.aya4net.nl). Participants $(n=66)$ were asked whether they were familiar with AYA care and if they were aware of the existence of the online AYA community; $59 \%(n=39)$ indicated they knew the community. Nine of 


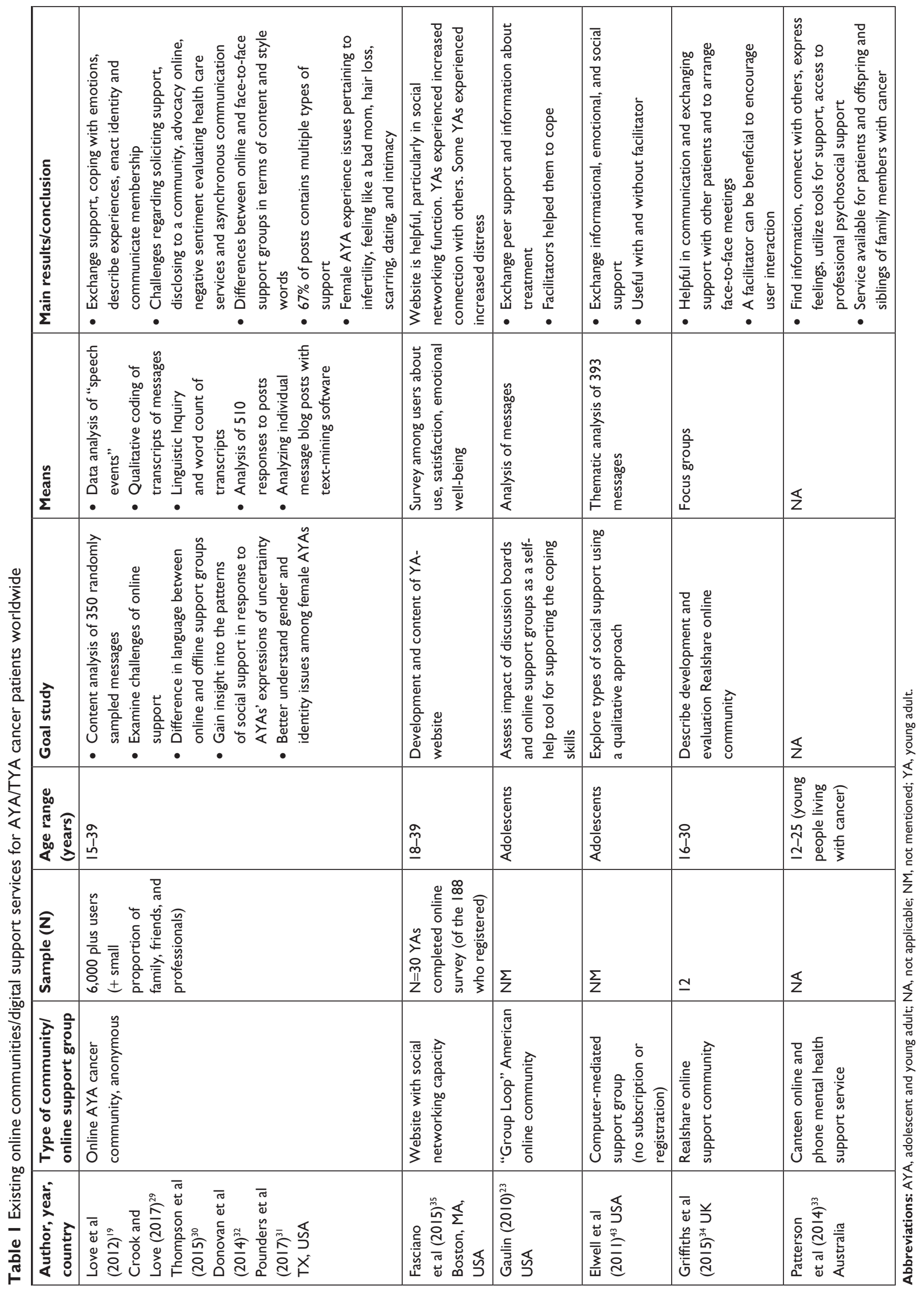


them were not a member of the community and were asked for their reasons of not being a member. Those patients who indicated to they were a member of the community $(n=30)$ were asked to answer questions about the usefulness of the community.

\section{Study 3: content analysis}

The content of the forum (discussions between AYA patients) contains privacy-sensitive personal information: names, diagnoses, and shared experiences. This type of information cannot be used for research purposes without the explicit consent of the authors - in this case, the AYA patients. ${ }^{21}$ Therefore, we asked the members permission to use the content of their forum messages through an opt-in procedure: we published a message on the forum in the first half of April 2016 in which we explained the aim of our study and asked the users for their consent to used their data in anonymized form. The data of patients who gave informed consent were anonymized according to the recommendations of King. ${ }^{22}$ An independent research assistant went through all messages in our sample manually and replaced all mentions of person names (except for names of caregivers) by the string “****". All anonymized messages by users who gave consent were brought together in a sample that we refer to as the anonymized sample.

A commonly used and well-studied methodology for investigating the psychological processes through language use is the Linguistic Inquiry and Word Count (LIWC). ${ }^{23}$ The LIWC analyzes texts for indicators of psychological processes that are important for psychologically processing of difficult experiences. ${ }^{24,25}$ The developers of the LIWC defined a set of linguistic and psychological categories that can be recognized by words in the text. For each category, they defined a set of words that are indicators for that category. For example, the word $m e$ is an indicator of the linguistic category "first person singular", and the word good is an indicator of the psychological category "positive emotions". We used the Dutch version of the LIWC consisting of 66 categories. ${ }^{26}$ There are five types of categories: 1) standard linguistic dimensions (eg, personal pronouns, first-person singular, and past-tense verbs); 2) psychological processes (eg, positive emotions, anxiety, humans); 3) relativity (eg, time and space); 4) personal concerns (eg, work, money, religion); 5) experimental dimensions (eg, swearing). The categories are organized hierarchically. For example, the main category "cognitive processes" under "psychological processes" has several subcategories, among which "insight", "inclusive", and "exclusive". Due to this hierarchy, a word can belong to more than one category. For example, the word $i k$ ("I") occurs in the category "pronoun" as well as the category "first-person singular".

The LIWC has been used before to distil these psychological processes from the content of online support communities. ${ }^{27,28}$ In this study, we did not analyze the language use of individual authors on the forum, but instead, we quantified the presence of LIWC categories in the anonymized sample as a whole. We implemented the LIWC using the word lists published for the Dutch version of the LIWC. ${ }^{26}$ Our LIWC script takes as input the complete text of our anonymized sample, and a dictionary of LIWC categories with the indicator words per category. The script splits the text in words, then looks up each word in the LIWC dictionary, and adds a count for the corresponding LIWC category. For example, each occurrence of the word $m e$ in the forum leads to a count for the category "first-person singular" and each occurrence of the word good in the forum leads to a count for the category "positive emotions". The output of the script is a count of indicator words occurring for each LIWC category. We sorted the categories by their frequencies of occurrence in order to analyze which linguistic and psychological categories are the most frequent in the forum. Results are depicted as relative word counts per category, which is the sum of the numbers of occurrences of all the words in the category divided by the total number of words in the sample.

\section{Results \\ User statistics}

As of November 2017, the community has 433 registered members with a mean age at diagnosis of 25.7 years (SD 4.9) of which $71 \%$ is female. Of these members, $18 \%$ were diagnosed with breast cancer, 17\% with lymphoma, 10\% with sarcoma, 7\% with leukemia, 7\% with testicular cancer, and $41 \%$ with other cancer types. The mean time since diagnosis when subscribing to the online AYA community was 2.7 years (SD 4.4), with a median time of 1 year; members were from 52 of the 91 hospitals in the Netherlands. In 2016, the online AYA community was visited 35,327 times. A visit is defined as a single online activity of a person at a certain time point by means of an electronic device. On average, a visitor was online for 1 minute and 23 seconds and looked at 2.47 pages per visit. The most frequently visited part of the community is the introduction page where AYA cancer patients introduce themselves by giving background information about their diagnosis, treatment trajectory, and daily-life problems because of cancer. 


\section{Evaluation}

Sixty-six patients answered AYA questionnaires and, of those, $39(59 \%)$ responded to have been informed about the AYA community; 30 (77\%) became members of the AYA community and used the community on a regular basis. The nine patients who did not become members indicated that they had no interest (three patients), thought it was not useful for them (one patient), indicated that the community was not available at the time they were diagnosed (two patients), or were too fearful to hear the stories of other patients (three patients). Of the 30 community members, 25 (83\%) were female. Of this group, the mean age at diagnosis was 25.6 years (SD 6.4, range 18-35) and mean age of questionnaire completion was 29.8 years (SD 5.3, range 22-39). The most frequent cancer diagnoses were breast cancer (20\%), lymphoma (17\%), and brain tumor (10\%). Nine (30\%) members rated the community as slightly useful and $18(60 \%)$ rated the community as highly useful; three (10\%) patients had no opinion. Use of the community resulted in acknowledgment of their problems (56\%) and the feeling of being supported as well as in having valuable contacts with peers (63\%). Almost half of the users felt less lonely, and 78\% experienced recognition in the cancer stories of other AYAs. In an open question, AYA patients indicated the strong willingness to do something for other patients as the main reason to be an active member of the community (Table 2).

\section{Content analysis using the LIWC}

Only 14 members of the online community provided consent for use of their messages in our content analysis. Together, these 14 members have posted 1,896 messages on 293 topics between February 2014 (the date the online AYA community became available as a national service) and June 2016. This is $44 \%$ (total messages, 4,332) of the total number of messages posted in this period, indicating that these 14 members are among the most active members of the forum. The mean number of messages posted by the included users is 135 (SD 103, range 5-386). We analyzed those 1,896 messages in our sample by using the LIWC categories. The total number of words in the sample is 108,881 ; the number of distinct words is 11,622 . Of these, 1,981 occur in one or more LIWC categories. Figure 1 shows the 20 most frequent LIWC categories in the sample, with their relative word counts. The most frequent LIWC category is "present tense". There are 13,888 occurrences of words from that category in our sample (eg, "is", "have”, "be", “am"). This gives a relative word count of $0.128(13,888 / 108,888)$ for the category "present tense".
Table 2 Answers to the evaluation questionnaire about the usefulness of the online AYA community among 30 members

\begin{tabular}{|c|c|}
\hline $\begin{array}{l}\text { Answers given about usefulness } \\
\text { about the community }\end{array}$ & $\begin{array}{l}\text { AYA community } \\
\text { members, } \mathbf{n}(\%)\end{array}$ \\
\hline I feel listened to & $9(33 \%)$ \\
\hline I get recognition/acknowledgment & $15(56 \%)$ \\
\hline I find recognition in the stories of peers & $21(78 \%)$ \\
\hline I do not feel lonely any more & $13(48 \%)$ \\
\hline I have good contact with peers & $17(63 \%)$ \\
\hline My questions are being answered & $9(33 \%)$ \\
\hline I feel more self-confident & $7(26 \%)$ \\
\hline $\begin{array}{l}\text { I get advice about coping with } \\
\text { problems }\end{array}$ & $12(44 \%)$ \\
\hline I make new friends & $12(44 \%)$ \\
\hline I feel reassured & $4(15 \%)$ \\
\hline I feel safe & $7(26 \%)$ \\
\hline I feel supported & $17(63 \%)$ \\
\hline Other & $\begin{array}{l}\text { - It gives me the opportunity } \\
\text { to share knowledge and } \\
\text { to help others. It appears } \\
\text { valuable to do something } \\
\text { in a hopeless situation } \\
\text { - I can support others } \\
\text { - It is good to notice that } \\
\text { I am not the only person } \\
\text { with problems }\end{array}$ \\
\hline
\end{tabular}

Abbreviation: AYA, adolescent and young adult.

\section{Discussion}

This study reports the use, evaluation, and content analysis of the online community for Dutch AYA cancer patients. To our knowledge, this is one of the first secure, closed communities for AYA cancer patients in Europe. Our online community is only accessible for AYA patients and survivors, in contrast with the large US community where family, friends, and professionals also have access. ${ }^{19,29-32}$ Similarly, the Australian community is also accessible for siblings and offspring of family members with cancer. ${ }^{33}$ In the United Kingdom and Australia, digital platforms focus on younger age groups (16-25 years ${ }^{34}$ and $12-24$ years, ${ }^{33}$ respectively) in comparison to the USA online support forum which serves AYA cancer patients between 15 and 39 years. All online communities have this in common that members provide and receive informational, emotional, and/or social support to some extent.

User statistics of AYA communities have never reported. The user statistics of our study showed that the most common tumor types were breast cancer and lymphoma and that members were most often women. This is in line with previous research where most members were female. ${ }^{34,35}$ It could be that men have less need for peer support and are, therefore, less likely to become a member, or prefer other 


\section{Relative word count for category in sample of AYA community}

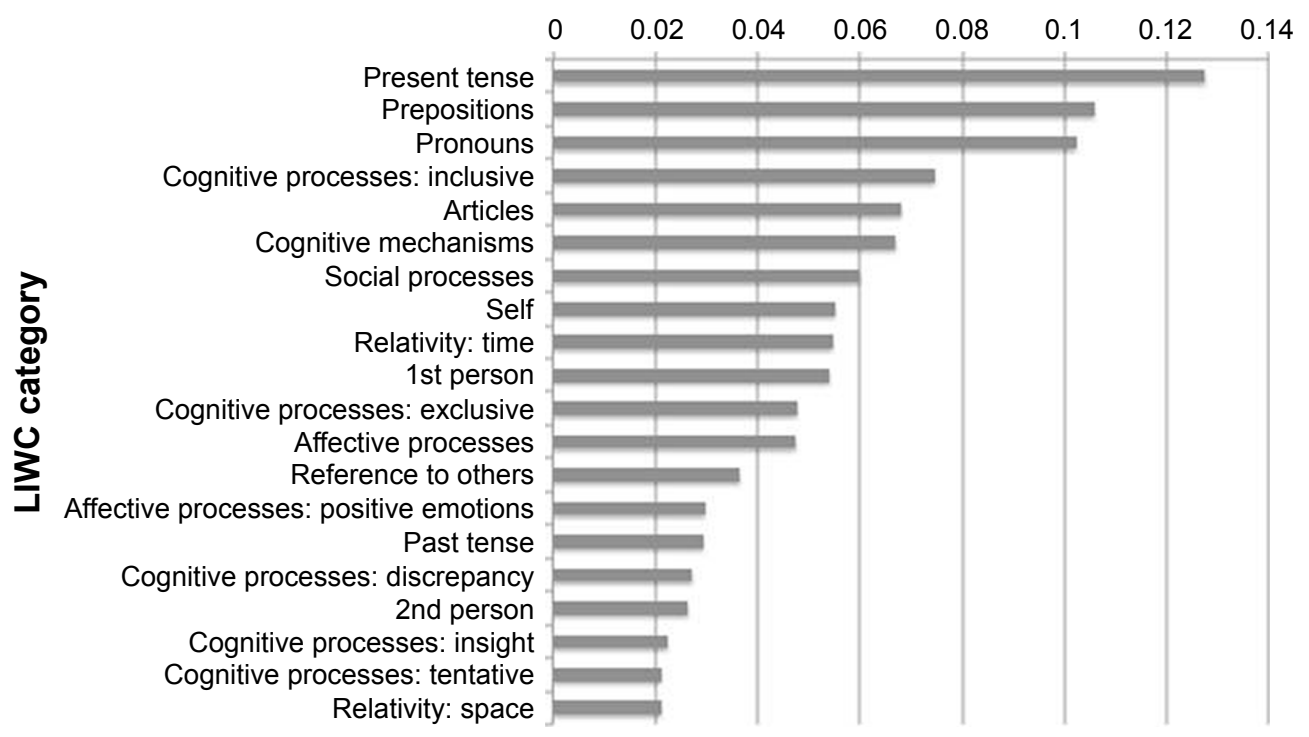

Figure I The 20 most frequent LIWC categories in the online AYA community sample, with their relative word counts. Abbreviations: AYA, adolescent and young adult; LIWC, Linguistic Inquiry and word count.

kinds of peer support, such as face-to-face interactions or via sporting activities. ${ }^{36}$ The short mean duration of online community visits may indicate that members use the community not as an extensive chat service but more as a forum to gain knowledge, express emotions, and get recognition. However, it might be an indication of the fact that AYA patients became scared of all that was written or that they did not like the online community and thought it to be something else. Thus, the results of the current study are hypothesis generating, and further in-depth research into the reasons for the short online duration is recommended.

More than half of the our study subjects rated the community as highly useful, especially with regard to the acknowledgment of feeling supported and the establishment of valuable contacts with peers. This is in line with the results of the content analysis showing that members of the online AYA community indeed find emotional and cognitive expression as well as emotional support. Given the theory of Ritterband, it could be hypothesized that higher levels of emotional and cognitive expression and emotional support may lead to better symptom control because this theory stated that internet interventions can lead to symptom improvement through mechanisms such as social support, transfer of knowledge, and feelings of recognition. ${ }^{10}$

The interpretation of the LIWC categories displays that the first three categories in Figure 1 are standard linguistic dimensions. Personal pronouns and especially references to self (I, me, and mine) are very common in discussion forum messages, indicating that the authors share narratives about themselves. ${ }^{37}$ The most interesting categories in Figure 1 are cognitive, social, and affective processes. The category "cognitive processes" includes the subcategories "inclusive", "exclusive", "discrepancy", "insight", and "tentative". Examples of words that belong to the category "inclusive" are; also, with, and completely, whereas examples of words of the category "exclusive" are; without, outside, and except. The category discrepancy covers words such as should, hope, must, and want, indicating a reality that is different than expected or wished for. The category "insight" contains words such as; find, see, and know, indicating insightful disclosure - a construct of empowerment that has been reported earlier for peer-directed patient support groups. ${ }^{28}$ The category "tentative" contains words such as; maybe, hope, and sometimes, indicating uncertainty. The category "social processes" covers words that describe interactions such as ask, people, and welcome. The category "affective processes" includes feelings and responses. In the online AYA community sample, positive emotions are the most prominent, with words such as; good, success, nice, happy, and better. The high frequency of words indicating cognitive, social, and affective processes may indicate that members of the online community find emotional support, emotional expression, and insight on the discussion forum.

In 2012, Love et al reported on the content analysis of messages in an open online AYA cancer support forum in 
the USA. They found that AYAs exchange emotional and informational support, cope with difficult emotions, use particular language to describe experiences, enact identity, and communicate membership on the online cancer support forum. ${ }^{19}$ Although we used another method to explore content, our results are largely in line with those of Love et al. In our study, we found that the majority of online discussions encompassed emotional support and emotional expression. Moreover, our results show that the community members gained more insight, expressed by words related to thinking, knowing, and considering. A difference between both studies, is that our content analysis was based on larger amount of messages than in the study of Love et al.

The main limitation of our study was the low participation rate of AYA community members that may limit the generalizability of our results. This may be explained by several factors. First, although in the Netherlands, every year, approximately 2,700 patients between age 18 and 35 are diagnosed with cancer, the AYA community currently has only 433 members. This might be attributable to the fact that patients and/or health care professionals are not familiar with AYA care and that the online AYA community only recently became available at the national level. Second, only a small part of the community members is active in discussions. This is in line with previous literature showing that only $10 \%$ of community members are active posters, the remaining $90 \%$ can be classified as "lurkers". 38 Third, as the online AYA community was developed 8 years ago and patients grow older, some early members may now have less need for peer support. Fourth, interviews with AYA cancer patients also revealed that the online AYA community was used to establish a first contact with peers and, thereafter, other faster social media such as WhatsApp were used to intensify the contact. Fifth, we cannot rule out selection bias, as patients recruited in the evaluation and the content analysis study might be the ones that are highly in need of peer support ("superusers") due to multiple health problems or are the patients who act as patient advocates.

The online AYA community is an example of an e-Health intervention that is highly valued by some users. E-Health has high confidentiality experience among cancer patients ${ }^{39}$ and has the potential to be cost-effective and to improve patient empowerment, ${ }^{40}$ psychological well-being, ${ }^{39,41}$ and health-related quality of life. ${ }^{41}$ Future studies should aim at in-depth knowledge about the use of the community in terms of not becoming a member of the community, reasons for stopping usage, reasons men visit the community less often, and whether additional elements should be added, in particular, to make it more attractive for men. Furthermore, it is worth exploring whether psychological interventions such as cognitive-behavioral therapy could be safely and effectively delivered online to AYA cancer patients. ${ }^{42}$ We expect that the AYA community will expand in terms of members and reputation in the future, because it only recently expanded from the regional to the national level.

\section{Conclusion}

The Dutch online AYA community facilitates peer support in a secure digital environment and, in particular, leads to AYAs with cancer expressing feelings, exchanging information, and coping better with cancer. Health care professionals should play an active role in drawing attention to the existence and the possible benefits of the online AYA community.

\section{Acknowledgments}

The authors would like to thank Robin Hooijer for his help with analyzing the Google Analytics data. The start of the Dutch online AYA community was supported by a grant from Alpe d'Huzes (grant no 2011-5346).

\section{Disclosure}

The authors report no conflicts of interest in this work.

\section{References}

1. Bellizzi KM, Smith A, Schmidt S; Adolescent and Young Adult Health Outcomes and Patient Experience (AYA HOPE) Study Collaborative Group, et al. Positive and negative psychosocial impact of being diagnosed with cancer as an adolescent or young adult. Cancer. 2012;118(20): 5155-5162.

2. Bibby H, White $\mathrm{V}$, Thompson $\mathrm{K}$, Anazodo A. What Are the Unmet Needs and Care Experiences of Adolescents and Young Adults with Cancer? A Systematic Review. J Adolesc Young Adult Oncol. 2017;6(1):6-30.

3. D'Agostino NM, Penney A, Zebrack B. Providing developmentally appropriate psychosocial care to adolescent and young adult cancer survivors. Cancer. 2011;117(10 Suppl):2329-2334.

4. Galán S, de la Vega R, Miró J. Needs of adolescents and young adults after cancer treatment: a systematic review. Eur J Cancer Care. Epub 2016 Aug 10.

5. Zebrack B. Information and service needs for young adult cancer patients. Support Care Cancer. 2008;16(12):1353-1360.

6. Stark D, Bielack S, Brugieres L, et al. Teenagers and young adults with cancer in Europe: from national programmes to a European integrated coordinated project. Eur J Cancer Care (Engl). 2016;25(3):419-427.

7. Ferrari A, Thomas D, Franklin AR, et al. Starting an adolescent and young adult program: some success stories and some obstacles to overcome. J Clin Oncol. 2010;28(32):4850-4857.

8. Slev VN, Mistiaen P, Pasman HR, et al. Effects of eHealth for patients and informal caregivers confronted with cancer: A meta-review. Int $J$ Med Inform. 2016;87:54-67.

9. Bright MA, Fleisher L, Thomsen C, Morra ME, Marcus A, Gehring W. Exploring e-Health usage and interest among cancer information service users: the need for personalized interactions and multiple channels remains. J Health Commun. 2005;10(Suppl 1):35-52.

10. Ritterband LM, Thorndike FP, Cox DJ, Kovatchev BP, GonderFrederick LA. A behavior change model for internet interventions. Ann Behav Med. 2009;38(1):18-27. 
11. Schiffman JD, Csongradi E, Suzuki LK. Internet use among adolescent and young adults (AYA) with cancer. Pediatr Blood Cancer. 2008; 51(3):410-415.

12. van Uden-Kraan CF, Drossaert CH, Taal E, Seydel ER, van de Laar MA. Participation in online patient support groups endorses patients' empowerment. Patient Educ Couns. 2009;74(1):61-69.

13. Fernsler JI, Manchester LJ. Evaluation of a computer-based cancer support network. Cancer Pract. 1997;5(1):46-51.

14. Mo PK, Coulson NS. Exploring the communication of social support within virtual communities: a content analysis of messages posted to an online HIV/AIDS support group. Cyberpsychol Behav. 2008;11(3):371-374.

15. van Uden-Kraan CF, Drossaert CH, Taal E, Shaw BR, Seydel ER, van de Laar MA. Empowering processes and outcomes of participation in online support groups for patients with breast cancer, arthritis, or fibromyalgia. Qual Health Res. 2008;18(3):405-417.

16. Setoyama Y, Nakayama K, Yamazaki Y. Peer support from online community on the internet among patients with breast cancer in Japan. Stud Health Technol Inform. 2009;146:886.

17. Piper LE. Generation Y in healthcare: leading millennials in an era of reform. Front Health Serv Manage. 2012;29(1):16-28.

18. Abrol E, Groszmann M, Pitman A, Hough R, Taylor RM, Aref-Adib G. Exploring the digital technology preferences of teenagers and young adults (TYA) with cancer and survivors: a cross-sectional service evaluation questionnaire. J Cancer Surviv. 2017;11(6):670-682.

19. Love B, Crook B, Thompson CM, et al. Exploring psychosocial support online: a content analysis of messages in an adolescent and young adult cancer community. Cyberpsychol Behav Soc Netw. 2012;15(10):555-559.

20. Frost J, Vermeulen IE, Beekers N. Anonymity versus privacy: selective information sharing in online cancer communities. J Med Internet Res. 2014;16(5):e126.

21. Wilkinson D. Thelwall M. Researching personal information on the public web: Methods and ethics. Social Science Computer Review. 2011;29(4):387-401.

22. King SA. Researching Internet Communities: Proposed Ethical Guidelines for the Reporting of Results. Inform Soc. 1996;12(2):119-128.

23. Gaulin, N. The experience of adolescents using online social networks to cope with their cancer: Exploring GroupLoop's discussion board and online support groups [digital dissertation]. Mervyn F and Massachusetts School of Professional Psychology; 2006. Available form: https://julac.hosted.exlibrisgroup.com/primo-explore/ search?query=any,contains, The $\% 20$ experience $\% 20$ of $\% 20$ adolescents $\% 20$ using $\% 20$ online $\% 20$ social $\% 20$ networks $\% 20$ to $\% 20$ cope $\% 20$ with $\% 20$ their\%20cancer:\%20Exploring $\% 20$ Grouploop $\% 27 \mathrm{~s} \% 20$ discussion $\% 20$ board $\% 20$ and $\% 20$ online $\% 20$ support $\% 20$ groups\&tab=er\&search_scope=HKU_ER\&vid=hku_er\&offset $=0$.

24. Pennebaker JW, Francis ME, Booth RJ. Linguistic Inquiry and Word Count: LIWC 2001. Mahway, NJ: Lawrence Erlbaum Associates; 2001.

25. Tausczik YR, Pennebaker JW. The Psychological Meaning of Words: LIWC and Computerized Text Analysis Methods. J Lang Soc Psychol. 2010;29(1):24-54.

26. Zijlstra H, van Meerveld T, van Middendorp H, et al. Dutch version of the Linguistic Inquiry and Word Count (LIWC), a computerized text analysis program. Gedrag \& Gezondheid: Tijdschrift voor Psychologie en Gezondheid. 2004;32(4):271-281.

27. Owen JE, Klapow JC, Roth DL, et al. Randomized pilot of a self-guided internet coping group for women with early-stage breast cancer. Ann Behav Med. 2005;30(1):54-64.
28. Lieberman M. The role of insightful disclosure in outcomes for women in peer-directed breast cancer groups: a replication study. Psychooncology. 2007;16(10):961-964.

29. Crook B, Love B. Examining the Light and Dark of an Online Young Adult Cancer Support Community. Qual Health Res. 2017;27(6):938-948.

30. Thompson CM, Crook B, Love B, Macpherson CF, Johnson R. Understanding how adolescents and young adults with cancer talk about needs in online and face-to-face support groups. J Health Psychol. 2016;21(11): 2636-2646.

31. Pounders K, Stowers K, Wilcox G, Love B, Mackert M. Exploring gender and identity issues among female adolescent and young adults who connect in an anonymous platform. $J$ Health Psychol. 2017;22(5):548-560.

32. Donovan EE, Lefebvre L, Tardif S, Brown LE, Love B. Patterns of Social Support Communicated in Response to Expressions of Uncertainty in an Online Community of Young Adults with Cancer. $J$ Appl Commun Res. 2014;42(4):432-455.

33. Patterson P, McDonald FE, Orchard P. A new Australian online and phone mental health support service for young people living with cancer. Australas Psychiatry. 2014;22(2):165-169.

34. Griffiths C, Panteli N, Brunton D, Marder B, Williamson H. Designing and evaluating the acceptability of Realshare: An online support community for teenagers and young adults with cancer. J Health Psychol. 2015;20(12):1589-1601.

35. Fasciano KM, Souza PM, Braun I, Trevino K. An Innovative Website in the United States for Meeting the Emotional and Supportive Care Needs of Young Adults with Cancer. J Adolesc Young Adult Oncol. 2015; $4(1): 44-49$.

36. Sawyer SM, McNeil R, McCarthy M, et al. Unmet need for healthcare services in adolescents and young adults with cancer and their parent carers. Support Care Cancer. 2017;25(7):2229-2239.

37. Morrow PR. Telling about problems and giving advice in an Internet discussion forum: some discourse features. Discourse Stud. 2006;8(4): 531-548.

38. Zhang S, O'Carroll Bantum E, Owen J, Bakken S, Elhadad N. Online cancer communities as informatics intervention for social support: conceptualization, characterization, and impact. $J$ Am Med Inform Assoc. 2017;24(2):451-459.

39. Leykin Y, Thekdi SM, Shumay DM, Muñoz RF, Riba M, Dunn LB. Internet interventions for improving psychological well-being in psycho-oncology: review and recommendations. Psychooncology. 2012;21(9):1016-1025.

40. Kuijpers W, Groen WG, Aaronson NK, van Harten WH. A systematic review of web-based interventions for patient empowerment and physical activity in chronic diseases: relevance for cancer survivors. $J$ Med Internet Res. 2013;15(2): 37.

41. Hong Y, Peña-Purcell NC, Ory MG. Outcomes of online support and resources for cancer survivors: a systematic literature review. Patient Educ Couns. 2012;86(3):288-296.

42. Mcgill BC, Sansom-Daly UM, Wakefield CE, et al. Therapeutic Alliance and Group Cohesion in an Online Support Program for Adolescent and Young Adult Cancer Survivors: Lessons from "Recapture Life". J Adolesc Young Adult Oncol. 2017;6(4):568-572.

43. Elwell L, Grogan S, Coulson N. Adolescents living with cancer: the role of computer-mediated support groups. J Health Psychol. 2011;16(2): 236-248.

\section{Dovepress}

\section{Publish your work in this journal}

Patient Preference and Adherence is an international, peer-reviewed, open access journal that focuses on the growing importance of patient preference and adherence throughout the therapeutic continuum. Patient satisfaction, acceptability, quality of life, compliance, persistence and their role in developing new therapeutic modalities and compounds to optimize

clinical outcomes for existing disease states are major areas of interest for the journal. This journal has been accepted for indexing on PubMed Central. The manuscript management system is completely online and includes a very quick and fair peer-review system, which is all easy to use. Visit http://www. dovepress.com/testimonials.php to read real quotes from published authors. 